\title{
Transcoding Method of H.264 Coded Bitstream for Interest Region
}

\author{
Ya-lin Wu and Soon-kak Kwon
}

\author{
Department of Computer Software Engineering, Dongeui University
}

\begin{abstract}
We propose a transcoding method of $\mathbf{H} .264$ coded bitsteam to control the picture quality dependently on the interest region. In the proposed method, first we find the model of quantization step-size and bitrate. Then a classification method according to the subjectively interest region within a video sequence is suggested. Also we propose a method that assigns a specific quantization step-size differentially according to the interest region within a video. In general, the subjective picture quality can be increased by applying the quantization step-size as a small value relatively for the interest region compared with the other regions. From the simulation, we show that the proposed method can make better subjective picture quality relatively in parts of interest region.
\end{abstract}

Index Terms-Transcoding method, Picture quality, Interest region, Quantization step-size

\section{INTRODUCTION}

THE conversion of the bitrates with keeping the same coding standard or syntax is widely used for various applications in the inter-network communications such as video streaming services.

When a final user cannot accept the higher bitrate of the originally compressed or/and stored bitstream, the bitrate reduction may be needed. The bitrate reduction algorithm converts from high bitrate bitstream to lower rate bitstream. There are two types of bitrate reductions [1-4]. The first one is full decoding and re-encoding. This method accomplishes a re-ordering of the reconstructed pictures and a re-estimation of motion vectors for the motion compensated coding, thus the complexity of implementation for the transcoding is increased. The second one is to achieve the target bitrate only with the re-quantization process in the transform domain. The bitstream is partially decoded only by both the inverse variable length code and inverse quantization. That is, the transformed coefficients are obtained from an inverse quantization with each quantization step-size. For reducing the video bitrate, the transformed coefficients are re-quantized with a larger quantization step-size. Finally, the coefficients generated by the re-quantization are converted into a new bitstream with a lower bitrate after the variable length code encoding process. This bitstream transcoding operation does not include the re-ordering and re-estimation for the motion compensation. Therefore, the bitstream transcoding by re-quantization process requires lower complexity and a little more processing time.

Manuscript received on March 31, 2008.

E-mail: skkwon@deu.ac.kr
For the bitstream transcoding, the several methods [5-9] have been proposed. All of methods concern with proper allocation of bitrate or quantization step-size in order to satisfy good picture quality or high PSNR (Peak Signal to Noise Ratio).

Also we have proposed trancoding method to control the picture quality dependently on the view focus [15]. When we watch the video, we would focus on the center position of the picture if the all of picture has the same priority. That is, it can be differently important of subjective picture quality dependently on the position of monitor which video plays, center and corner parts. It makes better video quality relatively in parts of center of monitor which can emphasize importance of visual quality relatively.

However, the interest regions are different to the video characteristics. Thus we classify the video into subjectively interest regions. Then the value of quantization step-size is differently assigned according to the location of slice of picture differently dependently on the classification of picture for the subjectively interest regions.

The organization of this paper is as follows. Section 2 provides the model of bitrate and quantization step-size. In Section 3, the proposed transcoding method is described for assign of the quantization parameters dependently on the interest regions. Section 4 shows the simulation results of the proposed method about quantization. In Section 5, we summarize the main results.

\section{MODEL OF BITRATE AND QUANTIZATION STEP-SIZE}

In the existing papers, the related formulas between bitrate and quantization step-size have been introduced, these are dependent on the quantizers and application areas.

For an MPEG-1,2 encoding system, we have proposed a formula based on a linear relation between bitrate $(R)$ and $\log$ function of a quantization step-size (Qstep) in a small range of bitrate [10].

$$
R=b+a \cdot \log \left(\frac{1}{Q \text { step }}\right)
$$

where, $b$ and $a$ are constants. Also, for an application to a wider range of bitrates, the above formula was improved as [11].

$$
R=b+\frac{a}{\text { Qstep }^{\gamma}}
$$

where, $0<\gamma \leq 2$. The other relative formula having two Qstep 
parameters was also proposed [9],

$$
R=\alpha \cdot Q \text { step }{ }^{-1}+\beta \cdot Q \text { step }{ }^{-2}
$$

where, $\alpha$ and $\beta$ are constants. Also, a linear relative formula was proposed as a logarithmic function between bitrate and quantization step-size [12],

$$
\log \text { Qstep }=b+a \cdot \log R
$$

where, $b$ and $a$ are dependent on the video characteristics.

The possibility of Eq. (4) was examined for H.264 [13] bitstream transcoding. JM9.5 [14] was used for the experiment and 4 videos were tried out for the proposed model relation. For video used in the experiment, flower garden, bus, foreman, waterfall were used and they had horizontal 352 pixels and vertical 288 pixels. The environment for the simulation did not include B picture, using H.264 baseline profile. 15 frames constitute one GOP and each video used the total 60 frames.

The relationship after requantization process was examined. In Fig. 1, the value of the quantization parameter for the bitstream before transcoding was set to 15 , in Fig. 2 to 25 . The Figures show the relation between the encoding rate and the re-encoded quantization step size when the values were increased by one.

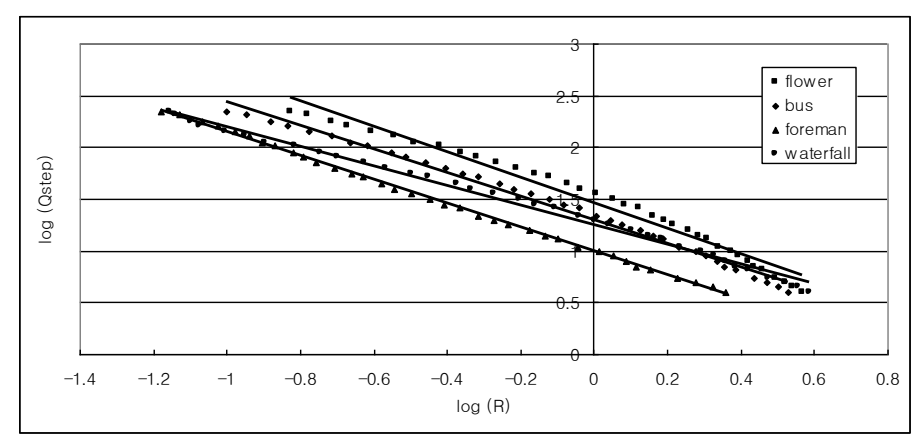

(a)

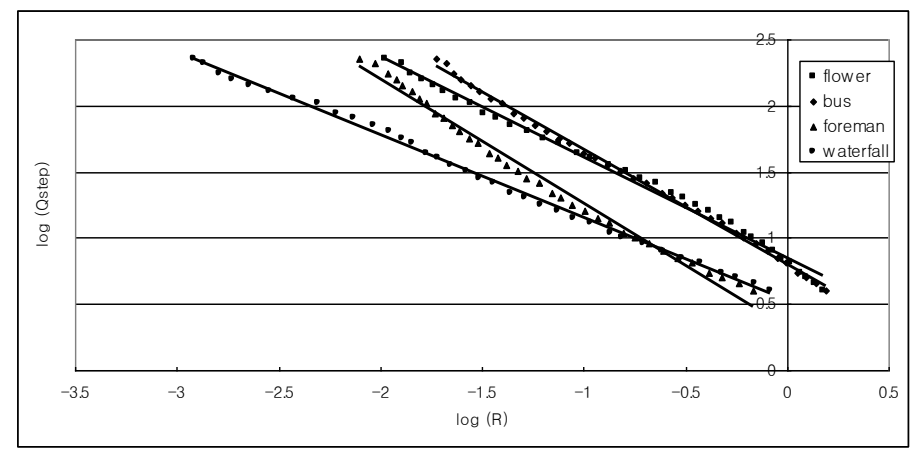

(b)

Fig. 1. Re-encoded R-Qstep relation when $\mathrm{QP}$ was increased by 1 on the video encoded with $\mathrm{QP}=15$ (Qstep=3.5), (a) I-picture (b) P-picture

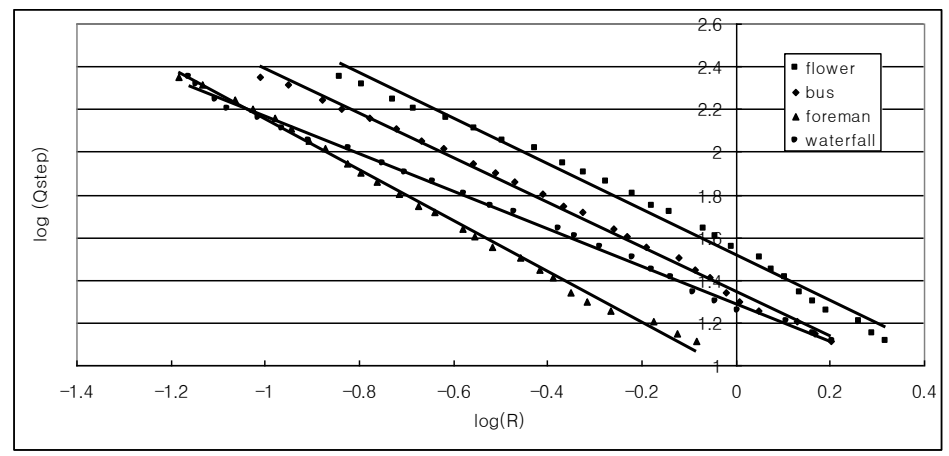

(a)

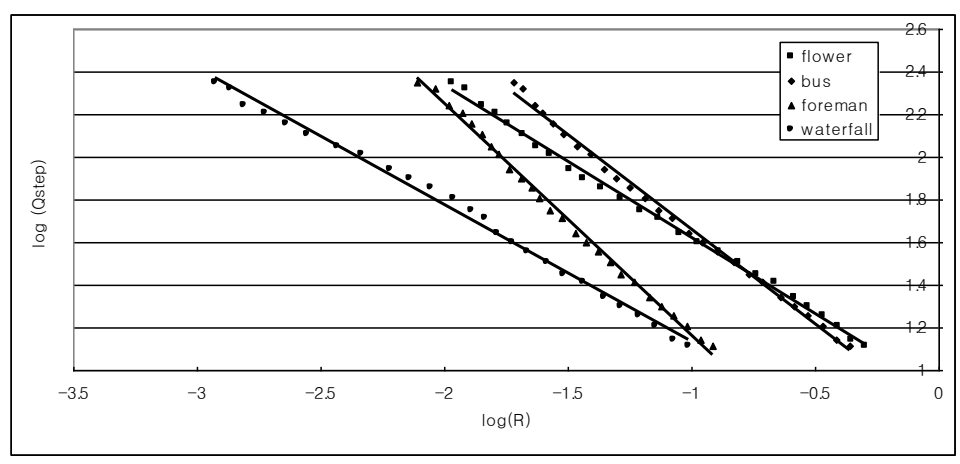

(b)

Fig. 2. Re-encoded R-Qstep relation when $\mathrm{QP}$ was increased by 1 on the video encoded with $\mathrm{QP}=25(\mathrm{Q} s t e p=1.1$ ), (a) I-picture (b) P-picture

\section{PROPOSED TRANSCODING METHOD FOR INTEREST REGION}

We have proposed the transcoding algorithm which updates the model parameters given for the previous picture or slice using an approximated relationship between bitrate and quantization step-size according to the coded picture-type [1]. That is, if a target bitrate in bits per second, $B R t(\mathrm{bps})$ is given in the case of already an encoded bitrate $B R_{l}(\mathrm{bps})$ in bits per second, then target quantization step-size Qstept is obtained from following equation.

$$
\text { Qstep }_{t}=\text { Qstep }_{1} \cdot\left(\frac{B R_{t}}{B R_{1}}\right)^{a}
$$

where, the value of $a$ is approximated by the previous coded results.

Also we have proposed to control the picture quality dependently on the view concern within displayed picture [15]. That is, it makes better video quality relatively in parts of center of monitor which can emphasize importance of visual quality relatively. In order to assign the value of quantization step-size differently according to the location of slice of picture, we found the following equation,

$$
\hat{Q}_{s}\left(i_{s}\right)=\alpha\left(i_{s}-\frac{N_{s}+1}{2}\right)^{2}+\bar{Q}_{s}-\alpha \frac{N_{s}^{2}-1}{12}, \quad 1 \leq i_{s} \leq N_{s}
$$


where, $i_{s}$ is an index of slice within a picture. $\hat{Q}_{s}\left(i_{s}\right)$ is a reassigned quantization step-size for $i_{s} . N_{s}$ is the total number of slice within a picture. $\alpha$ is a constant for control of the subjective picture quality. As the value of $\alpha$ is large, the difference of quantization step-sizes between the center slice and the top or bottom slice can be increased. Fig. 3 shows the reassigned quantization step-size for the different $\alpha$.

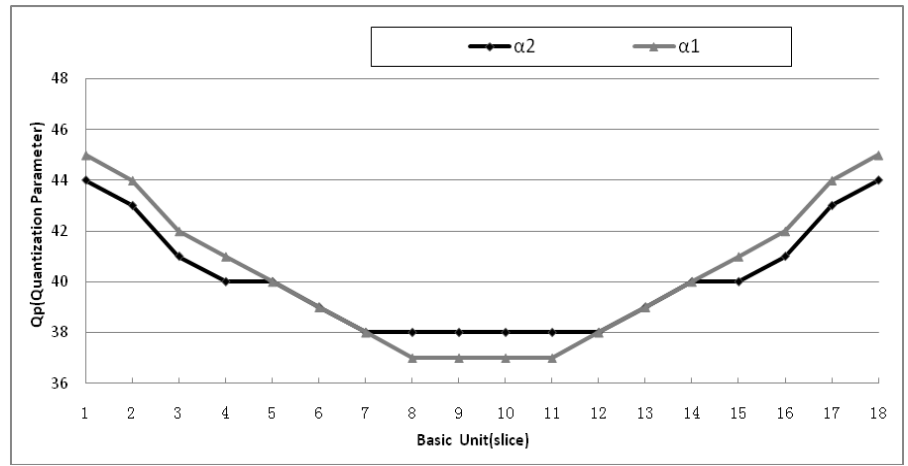

Fig. 3. Quantization step-size assigned dependently on the slice position according to Eq. (6), $\alpha_{1}>\alpha_{2}$.

In this paper we present the transcoding method to control the picture quality depended on the subjectively interest regions. The subjectively interest regions can be determined using motion vectors. Fig. 4 shows the motion vectors according to the characteristics of videos. The Bus video was made by moving a camera from right to left where motion vectors are presented in all macro blocks and represent similar vector values. Therefore, it is considered that the concerning is focused on the whole region of the video rather than certain specific regions. The Foreman video demonstrates the movement of the object located at the center of the video under the fixing of the background in which motion vector values are presented at the center of the video only. It shows that the concerning is focused on the central region that represents certain movements rather than that of the whole region of the video. Based on these characteristics, the current picture can be classified by following five different cases in the aspect of subjective importance.

Central focus picture: It shows motion vector values at the central region of a video in which the motion vector values at the contour regions are determined by ' 0 '

Contour focus picture: It shows motion vector values at the contour of a video like a zoom-out in a video in which the motion vector along the vertical direction represent large values.

Lower focus picture: It shows motion vector values at the lower region of a video only in which the motion vector values at the upper region are presented by ' 0 '.

Upper focus picture: It shows motion vector values at the upper region of a video only in which the motion vector values at the lower region are presented by ' 0 '.

Whole focus picture: In the case of the movement of the entire video like the video moved along the vertical or horizontal direction, it shows no concerning on a specific region but focuses the entire video.

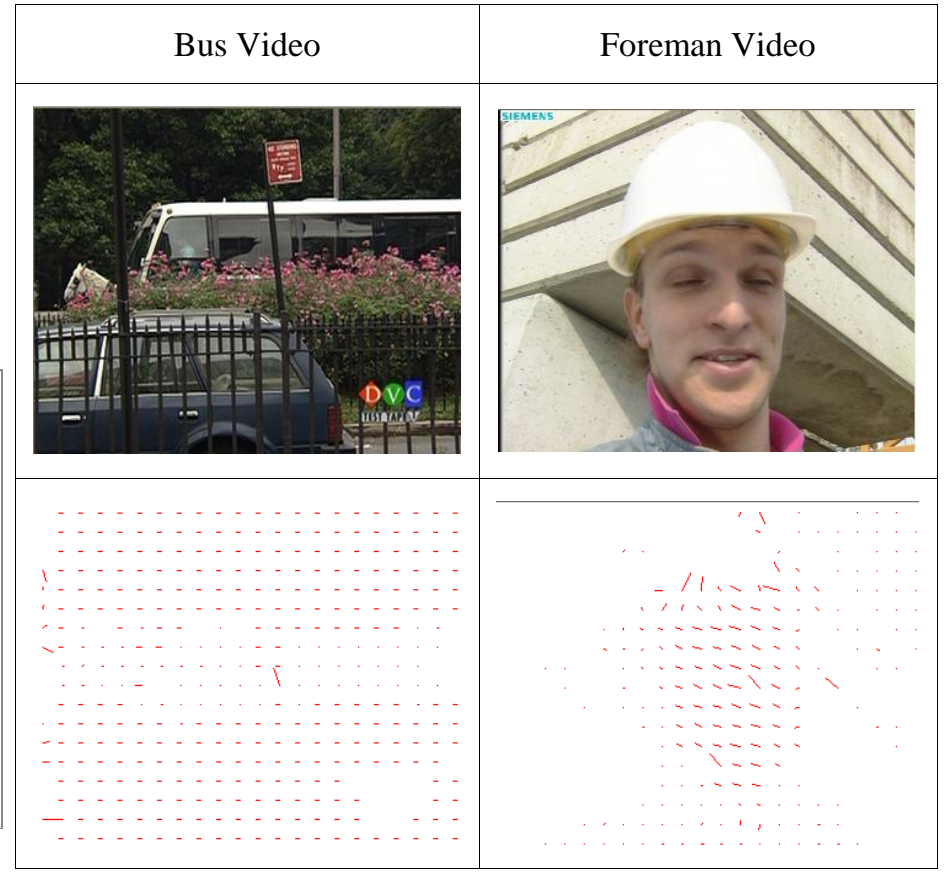

Fig. 4. Motion vectors for each video

After the above classification of picture for the subjectively interest regions, we assign the value of quantization step-size differently according to the location of slice of picture.

For the central focus picture, Eq. (6) is applied.

For the contour focus picture, a following equation is applied.

$$
\hat{Q}_{s}\left(i_{s}\right)=-\alpha\left(i_{s}-\frac{N_{s}+1}{2}\right)^{2}+\bar{Q}_{s}+\alpha \frac{N_{s}^{2}-1}{12}, \quad 1 \leq i_{s} \leq N_{s}
$$

\section{SimUlation Results}

For the simulation, JM 9.5 [14] of H.264 was used, and and four test sequences, 'Flower', 'Bus', 'Foreman', and 'Waterfall', have the same resolution of horizontal 352 pixels and vertical 288 pixels. The sequences were coded by the H.264 baseline profile, I-picture was repeated by a 15-picture interval within 30 pictures, and each picture contains 18 slices of the same size.

The simulation results for 'Foreman' sequence are shown in Fig. 5 to Fig. 8, where a bitstream, coded with the bitrate of $1.5 \mathrm{Mbps}$, is transcoded to the bitrate of $0.4 \mathrm{Mbps}$. 'Foreman' sequence is classified into the central focus picture, so Eq. (6) is applied to transcode. The quantization parameter obtained by the proposed transcoding method (denoted as Qp2) is compared with the before trancoding (denoted as Qp1). Also for comparison of effect of $\alpha$, four kinds of $\alpha$ are compared. In Fig. 5, $\alpha$ is set to give maximum difference of quantization parameter within one picture into 2 .

Fig. 9, Fig. 10, and Fig. 11 show the simulation results for 'Flower', Bus', and 'Waterfall' sequences, respectively. Similarly, the proposed method has the smallest values of the quantization parameter in the interest regions of the picture but largest values in the non interest regions. 


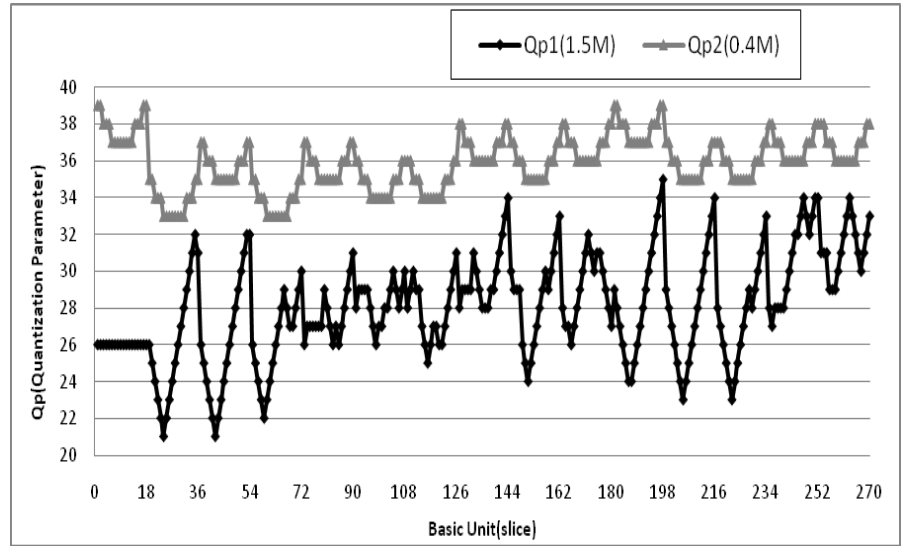

Fig. 5. Quantization parameters before and after transcoding for 'Foreman' test sequence, where $1.5 \mathrm{Mbps}$ bitstream (QP1) is transcoded to $0.4 \mathrm{Mbps}$ bitstream $\mathrm{Qp} 2(0.4 \mathrm{M})$. Maximum difference of quantization parameters within picture is set to 2 .

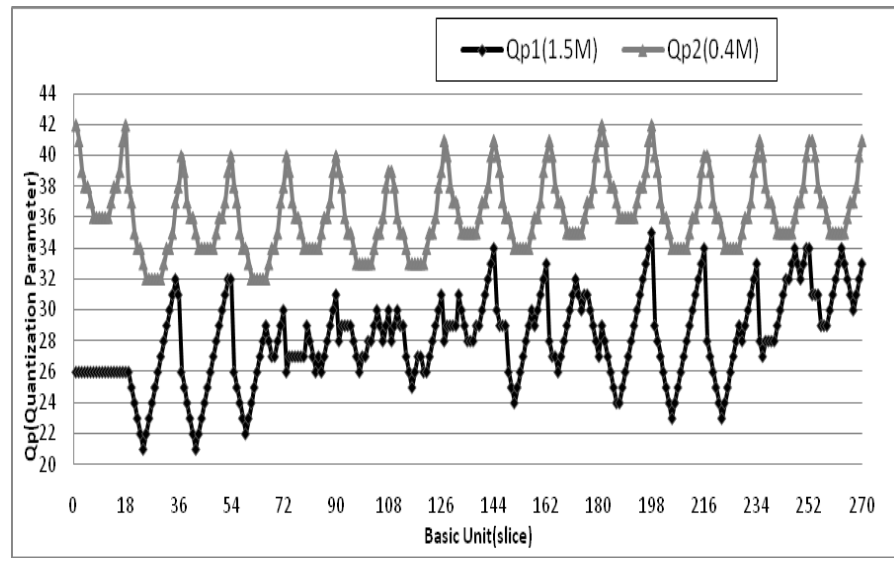

Fig. 6. Quantization parameters before and after transcoding for 'Foreman' test sequence, where $1.5 \mathrm{Mbps}$ bitstream (QP1) is transcoded to $0.4 \mathrm{Mbps}$ bitstream $\mathrm{Qp} 2(0.4 \mathrm{M})$. Maximum difference of quantization parameters within picture is set to 6 .

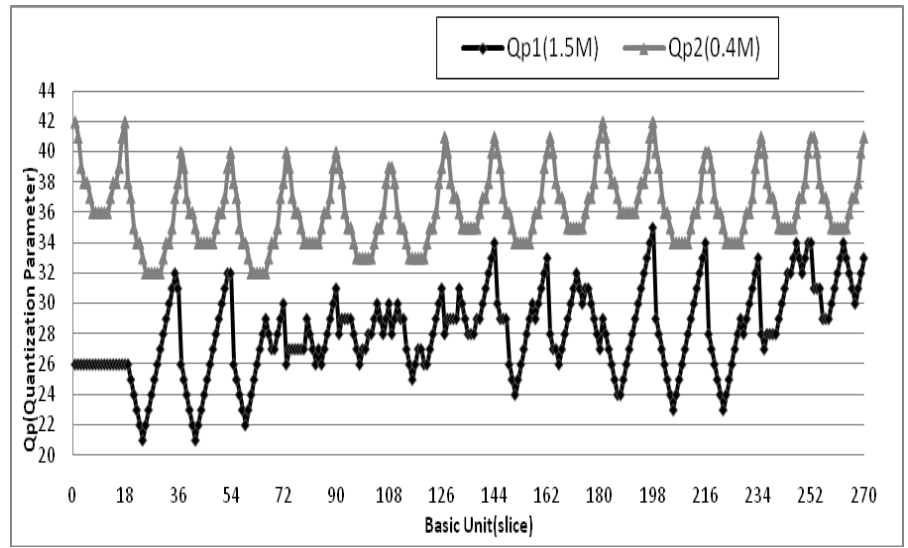

Fig. 7. Quantization parameters before and after transcoding for 'Foreman' test sequence, where $1.5 \mathrm{Mbps}$ bitstream (QP1) is transcoded to $0.4 \mathrm{Mbps}$ bitstream $\mathrm{Qp2}(0.4 \mathrm{M})$. Maximum difference of quantization parameters within picture is set to 8 .

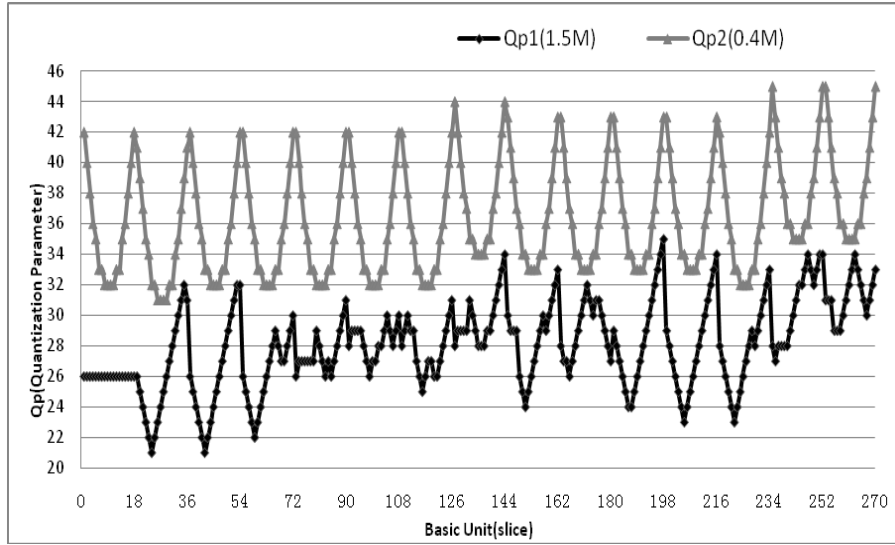

Fig. 8. Quantization parameters before and after transcoding for 'Foreman' test sequence, where $1.5 \mathrm{Mbps}$ bitstream $(\mathrm{QP} 1)$ is transcoded to $0.4 \mathrm{Mbps}$ bitstream $\mathrm{Qp2}(0.4 \mathrm{M})$. Maximum difference of quantization parameters within picture is set to 10 .

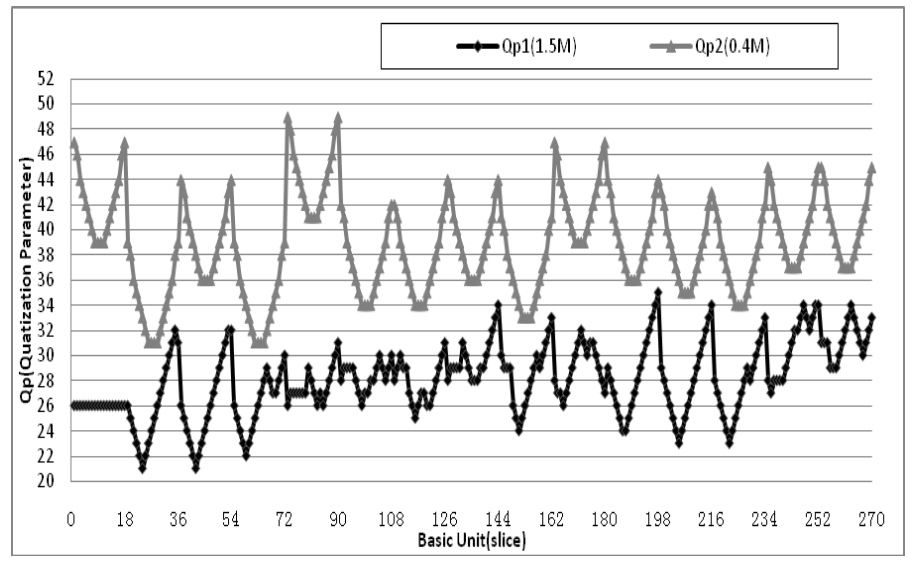

Fig. 9. Quantization parameters before and after transcoding for 'Flower' test sequence, where $1.5 \mathrm{Mbps}$ bitstream $(\mathrm{QP} 1)$ is transcoded to $0.4 \mathrm{Mbps}$ bitstream $\mathrm{Qp} 2(0.4 \mathrm{M})$. Maximum difference of quantization parameters within picture is set to 8 .

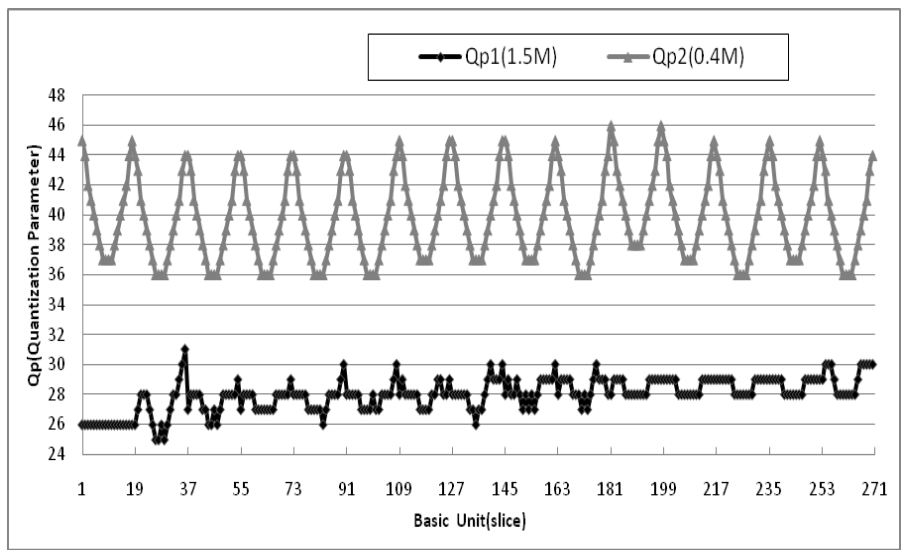

Fig. 10. Quantization parameters before and after transcoding for 'Bus' test sequence, where $1.5 \mathrm{Mbps}$ bitstream $(\mathrm{QP} 1)$ is transcoded to $0.4 \mathrm{Mbps}$ bitstream $\mathrm{Qp2}(0.4 \mathrm{M})$. Maximum difference of quantization parameters within picture is set to 8 . 


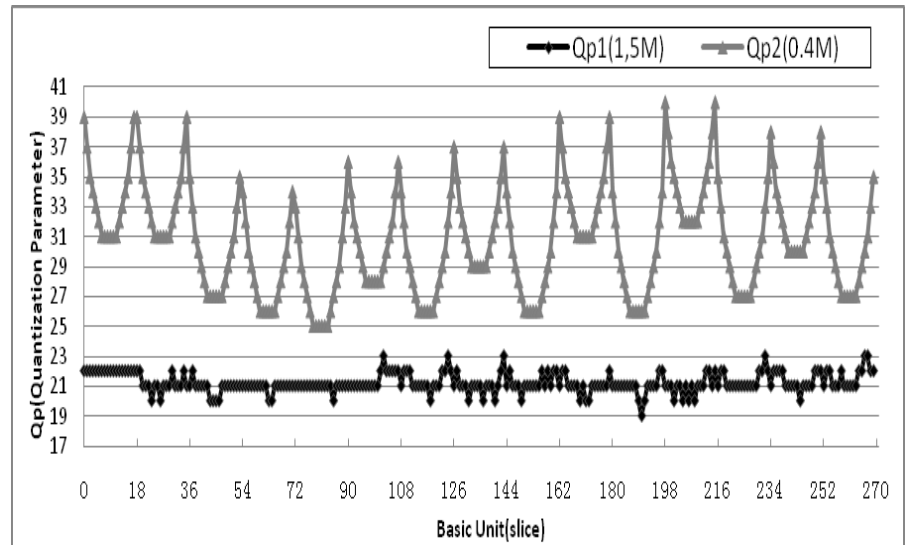

Fig. 11. Quantization parameters before and after transcoding for 'Waterfall' test sequence, where $1.5 \mathrm{Mbps}$ bitstream (QP1) is transcoded to $0.4 \mathrm{Mbps}$ bitstream Qp2(0.4M). Maximum difference of quantization parameters within picture is set to 8 .

Also, Fig. 12, Fig. 13, and Fig. 14 show the simulation results in the case of trancoded bitrate $0.8 \mathrm{Mbps}$ for 'Flower', Bus', and 'Waterfall' sequences, respectively.

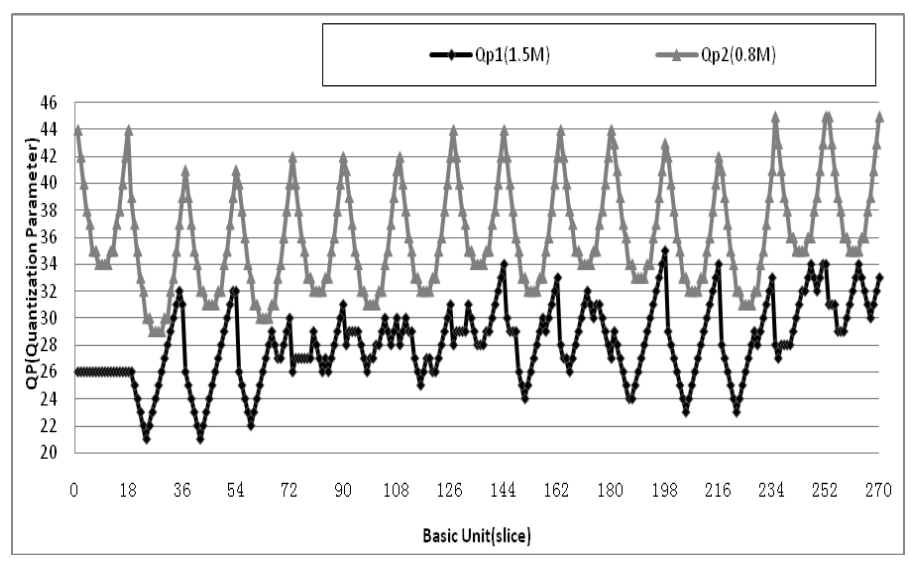

Fig. 12. Quantization parameters before and after transcoding for 'Flower' test sequence, where $1.5 \mathrm{Mbps}$ bitstream (QP1) is transcoded to $0.8 \mathrm{Mbps}$ bitstream $\mathrm{Qp} 2(0.8 \mathrm{M})$. Maximum difference of quantization parameters within picture is set to 8 .

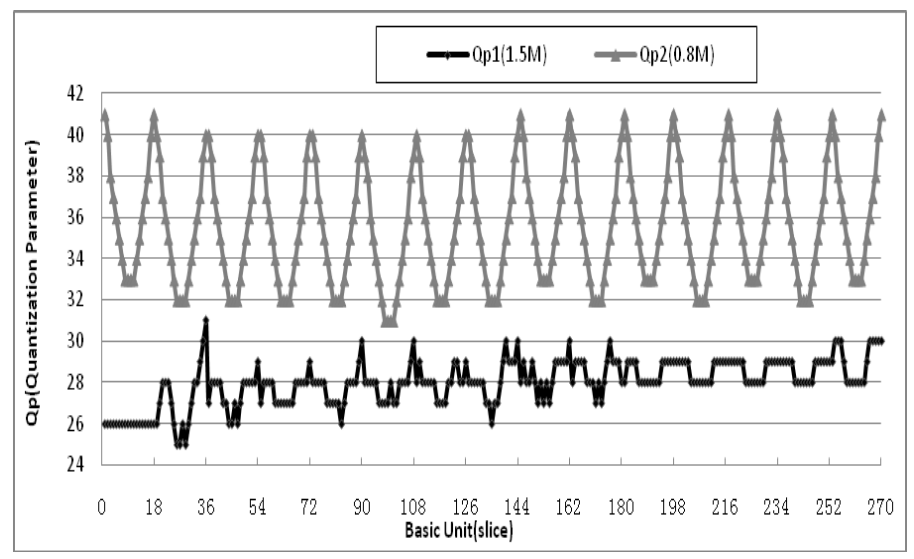

Fig. 13. Quantization parameters before and after transcoding for 'Bus' test sequence, where $1.5 \mathrm{Mbps}$ bitstream (QP1) is transcoded to $0.8 \mathrm{Mbps}$ bitstream $\mathrm{Qp} 2(0.8 \mathrm{M})$. Maximum difference of quantization parameters within picture is set to 8 .

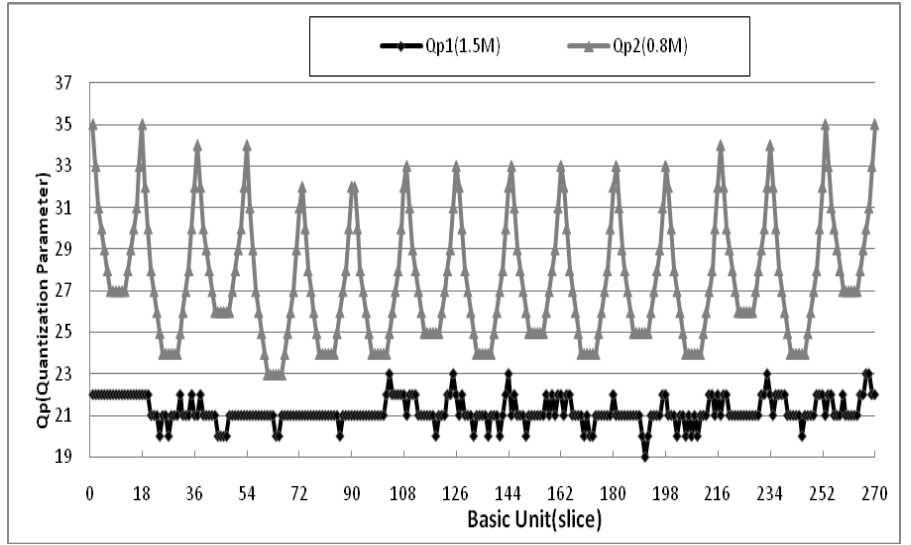

Fig. 14. Quantization parameters before and after transcoding for 'Waterfall' test sequence, where $1.5 \mathrm{Mbps}$ bitstream (QP1) is transcoded to $0.8 \mathrm{Mbps}$ bitstream Qp2(0.8M). Maximum difference of quantization parameters within picture is set to 8 .

\section{CONCLUSION}

This paper proposes a transcoding method that makes better video quality relatively in parts of interest regions. The interest regions are classified into five different cases such as central focus, contour focus, lower focus, upper focus, whole focus pictures. Then the smaller value of quantization step-size is assigned to more important position of interest focus but the larger value of quantization step-size is assigned to less interest focus. From the simulation, this paper illustrates that the target lower bitrate is obtained by the re-quantization process after finding the proper quantization step-size to control the picture quality dependently on the interest regions.

\section{REFERENCES}

[1] S.-k. Kwon, S.-w. Kim, J.-h. Lee, and J.-m. Lee, An Adaptive Transcoding Method, International Journal of Computer Science and Network Security, Vol. 8, No. 12, pp. 154-160, Dec. 2008.

[2] I.-M. Pao and M.-T. Sun, Encoding Stored Video for Streaming Applications, IEEE Trans. Circuits and Syst. Video Technology, Vol. 11, No. 2, pp. 199-209, Feb. 2001

[3] K. D. Seo, S. H. Lee, J. K. Kim, and J. S. Koh, Rate Control Algorithm for Fast Bit-rate Conversion Transcoding, IEEE Trans. Consumer Electronics, Vol. 46, No. 4, pp. 1128-1136, Nov. 2000.

[4] S. Acharya and B. Smith, Compressed Domain Transcoding of MPEG, Proc. IEEE Int. Conf. Multimedia Computing and Systems, Austin, Texas, pp. 295-304, July 1998 .

[5] M. Xia, A. Vetro, B. Liu, and H. Sun, Rate-distortion Optimized Bit Allocation for Error Resilient Video Transcoding, Proc. IEEE Int. Symp. Circuits and Systems, Vol. 3, pp. 945-948, May 2004.

[6] W. Lie, M. Tsai, and T. Lin, Rate-distortion Optimized DCT-domain Video Transcoder for Bit-rate Reduction of MPEG Videos, Proc. IEEE Int. Conf. Acoustics, Speech, and Signal Processing, Vol. 5, pp. 969-972, May 2004.

[7] L. Chen, L. Cheng, and S. Yu, Accurate Rate Control Method in Transcoding, Electron. Lett., Vol. 40, No. 1, pp. 16-18, Jan. 2004.

[8] J. Lei and N. Georganas, Accurate Bit Allocation and Rate Control for DCT Domain Video Transcoding, Proc. IEEE Canadian Conf. Electrical and Computer Engineering, Vol. 2, pp. 968-973, May 2002.

[9] H.-J. Lee, T. Chiang, and Y.-Q. Zhang, Scalable Rate Control for MPEG-4 Video, IEEE Trans. Circuit Syst. Video Technology, Vol. 10. No. 9, pp. 878-894, Sep. 2000.

[10] S.-k. Kwon, J.-k. Kim, and Y.-d. Park, Bit-rate Control for Uniform Picture Quality within Composed Video Sequence, Optical Engineering, Vol. 37, No. 11, pp. 3053-3060, Nov. 1998 
[11] W. Ding and B. Liu, Rate Control of MPEG Video Coding and Recording by Rate-quantization Modeling, IEEE Trans. Circuit Syst. Video Technology, Vol. 6, No. 1, pp. 12-20, Feb. 1996.

[12] J. Katto and M. Ohta, Mathematical Analysis of MPEG Compression Capability and its Application to Rate Control, Proc. Int. Conf. Image Processing, Washington, D.C., pp. 555-558, Oct. 1995.

[13] S.-k. Kwon, A. Tamhankar, and K. R. Rao, Overview of H.264/MPEG-4 Part 10, Journal of Visual Communications and Image Representation, Vol. 17, No. 2, pp. 186-216, Apr. 2006.

[14] H.264/AVC JM Software, http://iphome.hhi.de /suehring/ tml/download.

[15] S.-k. Kwon, H.-y. Lee, A Transcoding Method for Improving the Subjective Picture Quality, International Journal of Computer Science and Network Security, Vol. 9, No. 1, pp. 135-138, Jan. 2009.

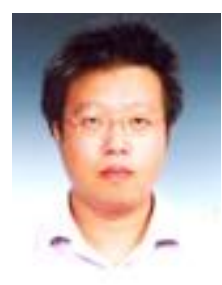

Ya-lin Wu completed the junior college curriculum from 2000 to 2003 in Department of Computer Media from Tian Jin University Of Technology, studied from 2003 to 2005 in Department of Mathematics from Tian Jin University. In 2008, he received the B.S degree in the Department of Computer Software Engineering of Dongeui University. Now he has been a master degree candidate. His research interests are in the areas of image processing, video coding, and video transmission.

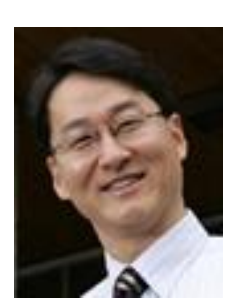

Soon-kak Kwon received the B.S. degree in Electronic Engineering from Kyungpook National University, in 1990, the M.S. and Ph.D. degrees in Electrical Engineering from Korea Advanced Institute of Science and Technology (KAIST), in 1992 and 1998, respectively. From 1998 to 2000, he was a team manager at Technology Appraisal Center of Korea Technology Guarantee Fund. Since 2001, he has been a faculty member of Dongeui University, where he is now an associate professor in the Department of Computer Software Engineering. From 2003 to 2004, he was a visiting professor of electrical engineering in the University of Texas at Arlington. His research interests are in the areas of image processing, video coding, and video transmission. 